\title{
A integralidade do SUS e a Judicialização por transplantes intestinais e multiviscerais
}

The integrality in the SUS and the judicialization by intestinal and multiple visceral transplants

La integridad del SUS y la legalización por los trasplantes intestinales y multiviscerales

Rodrigo Machado Santos ${ }^{1}$

RESUMO: A discussão sobre a judicialização da saúde no Brasil tem como tema central a questão do Sistema Único de Saúde - SUS não disponibilizar insumos e serviços de forma integral à população. Há demandas para transplantes de intestino e múltiplos órgãos, que em virtude do seu custo elevado, são combatidos pelos órgãos gestores do SUS, pelo custo elevado do procedimento. Tratou-se de um estudo de 4 casos ocorrido em 2014 sobre o tema para pacientes portadores de Síndrome de Berdon, Síndrome do Intestino Curto e a Doença de Inclusão Microvilositária. Concluiu-se pela pertinência da judicialização em vista da falta de recursos para os pacientes.

Palavras-chave: Judicialização. Integralidade. Transplante. Síndrome de Berdon. Síndrome do Intestino Curto.

ABSTRACT: The discussion about the Health Judicialization has a main topic that is the public system does not offer integrally services and inputs to the population. There are judicial demands for intestine and multiple organs transplants, but they are very expensive. There was a case study about 4 cases in Ministry of Health with intestine and multiple visceral transplant in patients who carry Berdon Syndrome, Short-Gut Syndrome and the Microvillous Inclusion Disease. It concluded for the relevancy of the judicialization for these poor patients.

Keywords: Judicialization. Completeness. Transplant. Berdon Syndrome. Short-Gut Syndrome.

RESUMEN: El debate sobre la judicialización de la salud en Brasil se centró en la cuestión de que el Sistema Único de Salud - SUS no está disponible en su totalidad al público. Hace demandas dirigidas a los trasplantes de intestino y de múltiples órganos, que, en virtud de su alto costo, son rechazadas por los órganos de gestión del SUS. Hizo un estudio de 4 casos teniendo como demandada el Ministerio de la Salud, sobre trasplantes de intestino y trasplantes multiviscerales para pacientes con Síndrome de Berdon, el Síndrome de intestino corto y la llamada Enfermedad de Inclusión Microvellosa. En este trabajo se analiza la cuestión de la integridad del Sistema Único de Salud, tratando de resolver el asunto que implica la prestación de cualquier tratamiento necesario a los pacientes del SUS.

Palabras-llave: Decisiones judiciales. Integridad. Trasplante. Berdon Síndrome. Síndrome del Intestino Corto.

${ }^{1}$ Advogado Email: rodrigomachadoadv@yahoo.com 


\section{Introdução}

Muito se tem discutido a respeito da Judicialização da saúde no Brasil, fenômeno estudado e discutido por todos os setores envolvidos, dentre eles a União, o Ministério Público e o Poder Judiciário, e encarado como prioritário por diversos atores que demandam e são demandados judicialmente. A base de toda esta discussão posta nas demandas judiciais se dá no âmbito do acesso à saúde de forma integral conforme referido na Constituição Federal (1), tendo como discussão principal o confronto entre o acesso à saúde versus a possibilidade da oferta do tratamento/insumo/medicamento pelo SUS.

A Lei Orgânica da Saúde no artigo 7º, II (2), afirma que a integralidade da assistência, deve ser entendida como um conjunto articulado e continuo de ações e serviços preventivos e curativos, individuais e coletivos, exigidos em todos os níveis do sistema.

A Constituição Federal de 1988 (1) abraça o direito à saúde e o direito de acesso à justiça, cuja construção contou com a pressão de diversos atores, entre eles movimentos sociais como também de juristas. Dallari (3) afirmava que a Constituição deveria prever meios de fácil acesso ao judiciário, além de facilitar a prolação de decisões mais justas e mais rápidas, ampliando-se assim o acesso de toda a população à justiça.

Vianna (4), entende por judicialização a valorização do poder judiciário como uma entidade que preencha um vazio sentimento de justiça. A judicialização é um mero indicador de que a justiça se tornou o último refúgio de um ideal democrático.

Capelletti (5) afirma que o sistema jurídico é o sistema pelo qual as pessoas podem reivindicar seus direitos e/ou resolver seus litígios sob os auspícios do Estado que, deve ser acessível a todos, deve produzir resultados que sejam individual e socialmente justos.

No presente trabalho foram estudados 4 (quatro) casos que foram levados à via judicial no ano de 2014, tendo como parte ré a União, cuja discussão central se dá na obrigatoriedade do Sistema Único de Saúde arcar com os custos dos transplantes de intestino e transplantes multiviscerais para pacientes portadores de síndromes que afetam os órgãos do trato intestinal como a Síndrome de Berdon, Síndrome do Intestino 
Curto e a chamada Doença de Inclusão Micovilositária, cujos custos ultrapassam a monta de US\$1.000.000,00 (um milhão de dólares), conforme dados fornecidos pelo Ministério da Saúde.

As três patologias descritas afetam diretamente na absorção dos nutrientes, causando diarreias constantes e falta de crescimento às pessoas acometidas por elas (6), tornando a vida dessas pessoas limitada, em especial com relação à alimentação e atividades diárias.

Para o tratamento dessas síndromes, o SUS disponibiliza conforme a portaria ํㅜ 120/2009 (7) do Ministério da Saúde, a nutrição via parenteral. Outra possibilidade de tratamento existente no meio médico e que não está disponível no SUS, é a realização de procedimentos cirúrgicos como o de transplante dos órgãos afetados, cuja técnica não é dominada ainda pelos profissionais médicos no Brasil.

O que se busca no presente trabalho é discutir a questão que permeia os debates jurídicos nesses casos. O SUS deve oferecer à população tudo o que possui de tratamentos disponíveis ou deve, independente da patologia ou da necessidade da população oferecer todo o tratamento necessário à sua cura?

A integralidade do Sistema Único de Saúde frente às demandas judicializadas visando cirurgia para as síndromes apresentadas deve ser considerada em seu sentido amplo ou por conta do valor elevado não deve ter o seu custeio através do sistema?

Tendo em vista a problemática, e a judicialização ser a única via de acesso para que a população tenha seus anseios atendidos, o trabalho analisa o conceito de integralidade aplicada ao SUS e o seu conceito trazido na legislação brasileira, em especial visando o atendimento das demandas por transplante de intestino e multivisceral.

\section{Metodologia}

Tratou-se de estudo das demandas judiciais propostas no ano de 2014 em desfavor da União, envolvendo pleitos por transplante de intestino e transplante múltiplo de órgãos em instituição particular no exterior. No total foram demandadas quatro ações, cujo relatório foi extraído do sistema de acompanhamento e controle de processos da Secretaria de Atenção à Saúde do Ministério da Saúde (SAS/MS). A partir 
das informações contidas no relatório, foi realizada consulta processual nos sítios eletrônicos do TRF-1 ${ }^{\text {a }}$ e TRF-3 ${ }^{\underline{a}}$, onde foram confirmados os andamentos dos referidos processos bem como foram extraídas as decisões judiciais proferidas nos mesmos.

Também foram realizadas pesquisas bibliográficas em livros, artigos científicos e teses nas bases de dados da biblioteca virtual em saúde - BVS, Lilacs e Google. A busca utilizou 4 (quatro) descritores: "integralidade no SUS", "acesso à saúde", "acesso à justiça"; e "Síndrome do Intestino Curto". Foram selecionadas no portal da legislação do governo federal leis vigentes que tem a saúde e o SUS como matéria, em especial a Constituição federal de 1988, a Lei 8080/90 e a lei 8132/90, tendo destaque os artigos que tratam do acesso da população ao SUS e da garantia do acesso à justiça.

Feita a leitura e seleção dos materiais encontrados passou-se a realizar pesquisa qualitativa utilizando-se a análise do conteúdo (8), sempre buscando na perspectiva do método aplicado encontrar as respostas necessárias ao questionamento formulado.

\section{Resultados}

Os casos estudados foram direcionados à Secretaria de Atenção à Saúde do Ministério da Saúde, que no ano de 2014 teve custos na ordem de $\mathrm{R} \$ 9.732 .120,77$ (nove milhões, setecentos e trinta e dois mil, cento e vinte reais e setenta e sete centavos).

Quadro 1. Demandas por transplante de Intestino e Multivisceral movidas contra a União Federal no ano de 2014

\begin{tabular}{|l|l|l|l|l|l|l|}
\hline & Sexo & Idade & Doença & Custo $(\mathrm{R} \$)$ & Realizou & Situação atual \\
\hline $\begin{array}{l}\text { AASO } \\
\text { A }\end{array}$ & $\mathrm{F}$ & 3 meses & Berdon & $2.106 .672,98$ & SIM & Óbito \\
\hline $\begin{array}{l}\text { CASO } \\
\text { B }\end{array}$ & $\mathrm{M}$ & 10 meses & Intestino Curto & 0,00 & NÃO & Óbito \\
\hline $\begin{array}{l}\text { CASO } \\
\text { C }\end{array}$ & $\mathrm{M}$ & 1 ano & $\underline{\text { Inclusão }}$ & $4.157 .610,24$ & NÃO & $\begin{array}{l}\text { Aguardando } \\
\text { doador }\end{array}$ \\
\hline $\begin{array}{l}\text { CASO } \\
\text { D }\end{array}$ & $\mathrm{M}$ & 15 anos & $\underline{\text { Intestino Curto }}$ & $3.467 .837,55$ & SIM & $\begin{array}{l}\text { Em } \\
\text { Recuperação }\end{array}$ \\
\hline
\end{tabular}

Fonte: Sistema de Judicialização da Secretaria de Atenção à Saúde do Ministério da Saúde 
O caso A foi proveniente de Sorocaba/SP, a autora por intermédio dos seus representantes legais, promoveu Ação Judicial no início de 2014, quando tinha apenas três (3) meses de vida pleiteando a realização de transplante multivisceral em no Hospital Jackson Memorial, em Miami-EUA.

A criança foi acometida da síndrome da Megabexiga, Microcólon e Hipoperistaltismo Intestinal (MMHI), também conhecida como Síndrome de Berdon. O Tribunal de Justiça do Estado de São Paulo (TJSP), determinou que a União procedesse por sua conta e diligências administrativas a transferência da paciente para Miami. Foi concedido um prazo de 15 dias e multa diária de $\mathrm{R} \$$ 100.000,00 (cem mil reais), na hipótese de descumprimento da decisão. O que foi prontamente atendido pelo Ministério da Saúde.

O transplante foi realizado quase um ano depois do encaminhamento da mesma tendo em vista que não foi encontrado de imediato um doador. Durante todo esse período a mesma foi mantida em nutrição parenteral. Contudo a paciente faleceu poucos meses depois da realização do transplante. $O$ montante financeiro para custear o tratamento somou $\mathrm{R} \$ 2.106 .672,98$ (dois milhões cento e seis mil, seiscentos e setenta e dois reais e noventa e oito centavos) depositados diretamente na conta do hospital americano e livremente geridos pelo mesmo, sem qualquer interferência do Ministério da Saúde ou mesmo sem qualquer comprovação de gastos no âmbito do processo judicial.

O caso B trata de um autor oriundo de Minas Gerais, que propôs a demanda quanto tinha 10 meses de vida, acometido de síndrome do intestino curto, pleiteando a realização de transplante no Hospital Jackson Memorial, em Miami-EUA. Após avaliação médica determinada pela justiça e que foi realizada por médico indicado pelo Ministério da Saúde, restou constatada a necessidade do transplante.

A Justiça Federal julgou procedente o pedido, determinando a imediata remoção do autor para os Estados Unidos. O Ministério da Saúde tomou todas as providências para o encaminhamento, contudo o paciente veio a óbito antes da viagem.

O caso C trata de Paciente oriundo da cidade de Franca - SP, que demandou judicialmente quando tinha pouco mais de 1 ano. Foi diagnosticado com a "doença de inclusão microvilositária" e por meio de ação judicial também pleiteou a realização de transplante de intestino no Hospital Jackson Memorial, em Miami-EUA. Na decisão de 
primeiro grau o M.M. juiz, antecipando os efeitos da tutela, determinou que a União providenciasse não só o custeio integral de tudo o que fosse necessário para que o autor fosse submetido à cirurgia de transplante de intestino e tratamentos respectivos junto ao Hospital de Miami, durante o tempo que se fizesse necessário, incluindo tratamento de homecare e inscrição em lista de espera Norte-Americana. Determinou ainda a instalação da família em local próximo ao nosocômio ou eventual alojamento dentro do próprio complexo hospitalar. Em cumprimento a determinação judicial o paciente após atingir o peso ideal para a realização do transplante no Hospital Samaritano em São Paulo foi encaminhado para o hospital americano onde ainda se encontra à espera de doador.

$\mathrm{O}$ caso $\mathrm{D}$ trata de Paciente de Curitiba/PR, que demandou à justiça quando tinha 15 anos de idade. Acometido da "Síndrome de Intestino curto", pleiteando realização de transplante de intestino no Hospital Jackson Memorial, em Miami-EUA. A decisão liminar em primeira instância lhe foi favorável, no sentido de determinar o encaminhamento do paciente para Miami, seguindo o precedente do caso A. O paciente foi transferido para o hospital em Miami e o transplante já foi realizado, estando atualmente realizando apenas exames de acompanhamento e segue em recuperação.

\section{Discussão}

O Ministério da Saúde em suas defesas apresentadas nos autos dos casos estudados pela Advocacia Geral da União argumenta que o maior problema da chamada Judicialização da saúde diz respeito à leitura meramente parcial do art. 196 da Constituição Federal e à relutância do Poder Judiciário em ler o referido artigo e os demais dispositivos constitucionais que regulam o direito à saúde por completo.

Segundo a Advocacia Geral da União, o dispositivo constitucional que inaugura a Seção que trata da saúde é muito claro ao vincular o direito à saúde a políticas públicas estatais, devendo-se resguardar, ainda, que o direito à saúde seja garantido a todos os cidadãos de forma igual. É isso que significa o atendimento universal e igualitário: que tudo o que estiver disponível no SUS esteja disponível a todos os cidadãos.

Carvalho e Santos (9) afirmam que a integralidade envolve o seu conceito ampliado e deve abranger tanto as ações assistenciais ou curativas quanto e 
prioritariamente as atividades de promoção da saúde e prevenção de doenças, contrariando, portanto, o argumento utilizado na defesa do Ministério da Saúde.

Não se trata de interpretação errônea do que dispõe a legislação, mas sim da interpretação que se tem dado quanto à obrigatoriedade do custeio do tratamento necessário à manutenção da vida dos pacientes.

Sarlet (10) ao discorrer sobre o direito à saúde afirma que o direito social que apresenta, simultaneamente, uma dupla dimensão defensiva e prestacional. Enquanto direito de defesa, o direito à saúde determina o dever de respeito, num sentido eminentemente negativo, ou seja, não afetar a saúde de alguém, mas, sim, preservá-la. $\mathrm{Na}$ dimensão prestacional, imputa o dever, em especial ao Estado, de executar medidas reais e concretas no sentido de fomento e efetivação da saúde da população circunstância que, neste último caso, torna o indivíduo, ou a própria coletividade, credores de um direito subjetivo a determinada prestação, normativa ou material.

A integralidade como eixo prioritário de uma política de saúde, ou seja, como meio de concretizar a saúde como uma questão de cidadania, significa compreender sua operacionalização a partir de dois movimentos recíprocos a serem desenvolvidos pelos sujeitos implicados nos processos organizativos em saúde: a superação de obstáculos e a implantação de inovações no cotidiano dos serviços de saúde, nas relações entre os níveis de gestão do SUS e nas relações destes com a sociedade (11).

Argumentar que não existe no Sistema Único de Saúde tratamento que atenda à necessidade do paciente é negar o propósito da concepção do próprio sistema.

A saúde é um direito fundamental do ser humano, devendo o Estado prover as condições indispensáveis ao seu pleno exercício (2) incluindo meios para a promoção de todo e qualquer tratamento necessário à manutenção da vida dos cidadãos, em detrimento de questões financeiras e orçamentárias.

A literatura médica aponta dois importantes tratamentos possíveis visando a cura ou a manutenção do bem-estar dos pacientes acometidos com as patologias, que resultam em falência intestinal, o transplante de intestino e a nutrição parenteral total.

A nutrição parenteral é coadjuvante ao tratamento específico da doença de base, como no caso dos pacientes desnutridos submetidos a cirurgias eletivas, podendo ser 
ainda considerado o tratamento primário como em casos de síndrome do intestino curto (12).

A indicação do transplante deve-se ter extrema cautela já que o transplante de intestino é proposta lógica para tratar a falência de intestino, porém existem grandes dificuldades relacionadas a esse procedimento. Problemas como rejeição de difícil controle, imunossupressão agressiva, infecções graves, técnica cirúrgica complexa, dificuldades na preservação do enxerto, doenças linfoproliferativas, entre outros, limitam a indicação usual do transplante (13).

Ainda assim, as chances de sobrevida dos pacientes acometidos dessas síndromes aumentaram nas últimas décadas, o desenvolvimento de novas drogas imunossupressoras, de modelos eficientes de imunossupressão, da terapia nutricional, dos cuidados intensivos e do controle de infecções melhorou significativamente os resultados na modalidade terapêutica do transplante (13).

Recentes avanços do Transplante Intestinal e Multivisceral causaram aumento da sobrevida e qualidade de vida em pacientes portadores de Falência Intestinal grave e com complicações da Nutrição Parenteral Domiciliar (13).

No Brasil, além de dois casos pioneiros realizados pelo Dr. Okumura no Hospital das Clínicas do Faculdade de Medicina da Universidade de São Paulo na década de 60, foram realizados mais recentemente um Transplante de Intestino na Santa Casa de São Paulo e três casos na Faculdade de Medicina de São José do Rio Preto, com maior sobrevida do enxerto de três meses e apenas um paciente em São José do Rio Preto obteve sobrevida prolongada após a retirada do enxerto intestinal transplantado. Infelizmente não existem atualmente serviços ativos de transplante intestinal no Brasil (13).

A Coordenação Geral do Sistema Nacional de Transplantes-CGSNT/SAS/MS traçou um diagnóstico da situação do serviço no Brasil e afirma que é muito recente na literatura médica brasileira a realização de transplantes desses órgãos, tendo sua realização iniciada efetivamente em 2012 (14).

O baixíssimo número de transplantes dessa modalidade realizados até agora no Brasil no total de seis procedimentos, do ano de 2012 até 2015 ainda não possibilitou que os profissionais brasileiros adquirissem a expertise necessária (14). Além disso, 
essa modalidade de transplante ainda não está regulamentada, o Regulamento Técnico do Sistema Nacional de Transplantes vigente (Portaria GM 2.600/2009) não contempla essa terapia (14).

A revisão de todo Regulamento está sendo feita pela Coordenação Geral do Sistema Nacional de Transplantes (CGSNT) com o apoio das Câmaras Técnicas Nacionais, que deliberam sobre as políticas de transplantes de órgãos, sendo que todos os módulos, incluindo o de intestino e multivisceral, ainda carecem de ajustes (14).

Importante frisar que o Ministério da Saúde, já vem pensando uma alternativa ao tratamento desses pacientes, visando o atendimento dos próximos casos que demandarem pelo transplante de intestino ou multivisceral, buscando a promoção de Cooperação com centros mais avançados que realizam esse procedimento com vasta experiência e sucesso. A cooperação teria como objetivo principal qualificar os serviços, possibilitando que as equipes adquiram experiência e segurança de modo que, em médio/longo prazo se tornem autônomos e possam atender, no Brasil, qualquer cidadão que necessite dessas modalidades de transplantes (14).

Enquanto a medicina não evolui para a solução dessas patologias, os seus pacientes têm todo o direito de serem tratados com amplo acesso aos tratamentos disponíveis. Seja utilizando o transplante ou a nutrição específica, devendo ficar à cargo do seu médico a indicação do tratamento correto a ser aplicado.

\section{Conclusão}

Diante da problemática apresentada, conclui-se que a judicialização é fruto de um aumento da participação social da população, seja exigindo direitos seja cobrando dos atores políticos ações e garantias constitucionais.

Frise-se que os pacientes dos casos estudados somente buscaram a via judicial porque não foi encontrado no Sistema Único de Saúde nem no Brasil o tratamento que necessitavam e a alternativa terapêutica existente, não visa a cura das suas patologias.

Argumentar que o que ocorre no país é uma interpretação errônea do que diz a Constituição Federal, é se eximir do dever de oferecer saúde à população. $\mathrm{Na}$ concepção do SUS, se pensou em um sistema de saúde público, de acesso à todos e sem distinção, cabendo aos gestores desenvolverem mecanismos para o atendimento. 
Não há que se ter dúvidas quanto a forma de como deve ser tratada a questão da integralidade do SUS. Ela deve ser encarada sempre no seu sentido amplo, onde o direito à vida e o direito à saúde são mais importantes que qualquer questão econômica ou orçamentária.

As demandas por transplante de intestino e por múltiplos órgãos surgem em virtude da necessidade daquele tipo de tratamento, devendo assim todo o cuidado e atendimento ser disponibilizados aos que dele necessitam.

Questões orçamentárias devem ser resolvidas de maneira conjunta entre 0 legislativo e o executivo, visando sempre o fomento de políticas públicas para atender à população de forma integral.

A União deve criar mecanismos que melhor possibilitem o desenvolvimento de novas tecnologias, buscando parcerias ou até mesmo formalizando convênios que envolvam a capacitação técnica de equipes brasileiras, para que o sistema atenda de maneira satisfatória a todos que dele necessitem.

Assim, o que deve existir é uma ampliação do conceito de integralidade em toda a rede pública de saúde, estimulando ações, equipando melhor os centros de tratamento e capacitando cada vez mais as equipes médicas que estão dentro do SUS como fora dele, tendo em vista a ausência da terapêutica no Brasil, para tornar viável a realização desse tipo de transplante no Brasil. Prevalecendo sempre o acesso amplo, irrestrito e integral à saúde.

\section{Referências}

1 Brasil. Constituição da República Federativa do Brasil, de 5 de outubro de 1988. [Acesso em 05 nov 2015]. Disponível em: http://www.planalto.gov.br/ccivil 03/Constituicao/Constituicao.htm

2 Brasil. Lei 8080, de 19 de setembro de 1990. Dispõe sobre as condições para a promoção, proteção e recuperação da saúde, a organização e o funcionamento dos serviços correspondentes e dá outras providências.[Acesso em 05 nov 2015].

Disponível em http://www.planalto.gov.br/ccivil 03/Leis/L8080.htm

3 Dallari SG. O direito à saúde. Rev. Saúde Pública, 1988; 22(1):57-63.

4 Vianna LW, Carvalho MAR, Melo MPC, Burgos MB. A judicialização da política e das relações sociais no Brasil.1aㅡ ed. Rio de Janeiro:Revan;1999,25. 
5 Cappelletti M. Garth B. Acesso à Justiça. Ellen Gracie Northfleet. (Trad) Porto Alegre: Fabris, 1988.

6 Behrman RE, Kliegman RM, Jenson HB. Nelson tratado de pediatria. $17^{a}$ ed. Rio de Janeiro: Elsevier; 2005.

7 Brasil. Ministério da Saúde. Portaria no 120, de 14 de abril de 2009. Aprova as normas de classificação e credenciamento/Habilitaçao dos serviços de alta complexidade em terapia nutricional enteral e enteral/parenteral .[Acesso em 12 nov 2015]. Disponível em:http://bvsms.saude.gov.br/bvs/saudelegis/sas/2009/prt0120 1404 2009.html

8 Campos CJG. Método de análise de conteúdo: ferramenta para a análise de dados qualitativos no campo da saúde. Rev Bras Enfermagem Brasilia (DF) 2004 set/out;57(5):611-4.

9 Carvalho G.I, Santos L. Sistema Único de Saúde: Comentários à Lei Orgânica de Saúde (Leis n. 8080/90 e 8142/90). Campinas: Editora Unicamp, 2002; 3.33-53.

10 Sarlet, I apud Figueiredo. Mariana Filchtiner. Direito fundamental à saúde: parâmetros para sua eficácia e efetividade. Porto Alegre: Livraria do Advogado; 2007. 88.

11 Pinheiro R., Mattos R. Construção Social da Demanda: direito à saúde, trabalho em equipe e participação em espaços públicos. 1.ed. Rio de Janeiro: Cepesc/MS/Uerj/ Abrasco; 2005.

12 Borges RM, Maduro IPN, Chueire FB, Fernandes SL, Soriano EA, Suen VMM. Et al.Terapia Nutricional Parenteral. Diagnóstico e Tratamento. $1^{1 \stackrel{a}{ }}$ ed. Barueri/SP:Manole;2006. 452-459.

13 Galvão F. Lee A. Pécora, A., David A. Pugliesi V. Tannuri U. D'albuquerque, K.. Tratamento da Falência Intestinal Complicada. A era do Transplante Intestinal. WNutrology, North America, 4, aug. 2011.[Acesso em 12 nov 2015]. Disponível em: http://abran.org.br/RevistaE/index.php/IJNutrology/article/view/24/20.

14 Borba HM., Passos JEF (Departamento de Atenção Especializada e Temática, Secretaria de Atenção à Saúde, Ministério da Saúde). Nota Técnica 072/2015. 07 de outubro de 2015.

Como citar este artigo:

Machado Rodrigo. A integralidade do SUS e a Judicialização por transplantes intestinais e multiviscerais. Revista Cadernos Ibero-Americanos de Direito Sanitário. 2016 abr./jun, 5(2):131-141. 